\title{
Information-Guided Surgery of Intracranial Gliomas: Overview of an Advanced Intraoperative Technology
}

\author{
Yoshihiro Muragaki ${ }^{1,2^{*}}$, Mikhail Chernov ${ }^{1,2}$, Kitaro Yoshimitsu ${ }^{1}$, \\ Takashi Suzuki ${ }^{1}$, Hiroshi Iseki ${ }^{1,2}$, Takashi Maruyama ${ }^{1,2}$, Manabu Tamura ${ }^{1,2}$, \\ Soko Ikuta1 ${ }^{1}$, Masayuki Nitta ${ }^{1}$, Atsushi Watanabe ${ }^{1}$, Taiichi Saito ${ }^{1,2}$, \\ Jun Okamoto ${ }^{1}$, Chiharu Niki ${ }^{1}$, Motohiro Hayashi ${ }^{1,2}$ and Kintomo Takakura ${ }^{1,2}$ \\ ${ }^{1}$ Faculty of Advanced Techno-Surgery and ${ }^{2}$ Department of Neurosurgery, \\ Tokyo Women's Medical University, Tokyo, Japan
}

Submitted November 2011. Accepted for publication August 2012.

\begin{abstract}
The objective of this paper is to provide an overview of advanced information-guided surgical management technology of intracranial gliomas implemented in Japan, represented by the Intelligent Operating Theater at the Tokyo Women's Medical University, established in 2000, where more than 1000 patients with various intracranial pathology have received neurosurgical treatment with application of the intraoperative MRI, updated neuronavigation, and advanced neurophysiological and histopathological monitoring. Additionally, a dedicated computer-based system for accumulating, storing, integrating, and analyzing various surgical data was developed, which provided an opportunity for adequate risk management and prediction of the surgical results. Application of these modalities and tools led to significant improvements in both surgical outcome and long-term patients' prognosis. Further technological developments, such as new therapeutic robot, dedicated laser, and high intensity focused ultrasound are currently under investigation. The final goal is to integrate all these devices and systems into comprehensive neurosurgical framework.
\end{abstract}

Keywords: glioma, neuronavigation, intraoperative MRI, awake craniotomy, surgical robot

\section{INTRODUCTION}

To date the clinical decision making is significantly influenced by the personal intuition and previous experience of the doctor. Experienced neurosurgeon is able to simulate the whole upcoming surgery in mind and to perform it on patient precisely and efficiently.

*Corresponding author: Yoshihiro Muragaki, Faculty of Advanced Techno-Surgery, Tokyo Women's Medical University, 8-1 Kawada-cho, Shinjuku-ku, Tokyo, Japan 162-8666. Phone: +81-3-3353-8111 (ext. 66003). Fax: +81-3-5321-1844. E-mail: ymuragaki@abmes.twmu.ac.jp. Other authors: m_chernov@abmes.twmu. ac.jp; kitaro@abmes.twmu.ac.jp; takashi_suzuki@abmes.twmu.ac.jp; hiseki@abmes.twmu.ac.jp; tmaruyama@nij.twmu.ac.jp; manabu97@abmes.twmu.ac.jp; sikuta@abmes.twmu.ac.jp; masayukin@nij.twmu.ac.jp; awatanabe@abmes.twmu.ac.jp; taiichis@nij.twmu.ac.jp; j-okamoto@abmes.twmu.ac.jp; chiharu-niki@ abmes.twmu.ac.jp; GKRmoto@gmail.com; ktakakura@abmes.twmu.ac.jp. 
This is remarkably facilitated with the advances of the modern neuroimaging and intraoperative neuronavigation. At present, various diagnostic data obtained with MRI, PET, digital angiography etc. may not only be visually inspected and analyzed, but coregistered, fused, and incorporated into computer-based navigation devices. Nevertheless, usually it is not the various images, but the training of the surgeon, that provides effective orientation in the surgical field, evaluation of the functional importance of the various anatomic structures, and estimation of the optimal resection of the lesion taking into account possible positive and negative consequences of the various intraoperative manipulations and actions. Therefore, to date the complex neurosurgical procedure, removal of glioma in the eloquent brain area for example, represents more an art than science or technique.

It can be expected, however, that in the third millennium the practical medicine will be transformed into a "feed-forward" process with scientifically-based prediction of the various risks and their preemptive management [1]. In fact, objectivity and reproducibility required for the surgical procedure in modern times can be provided by the medical engineering technology. This is illustrated in Figure 1, through the development of the specific equipment for precise visual assessment of

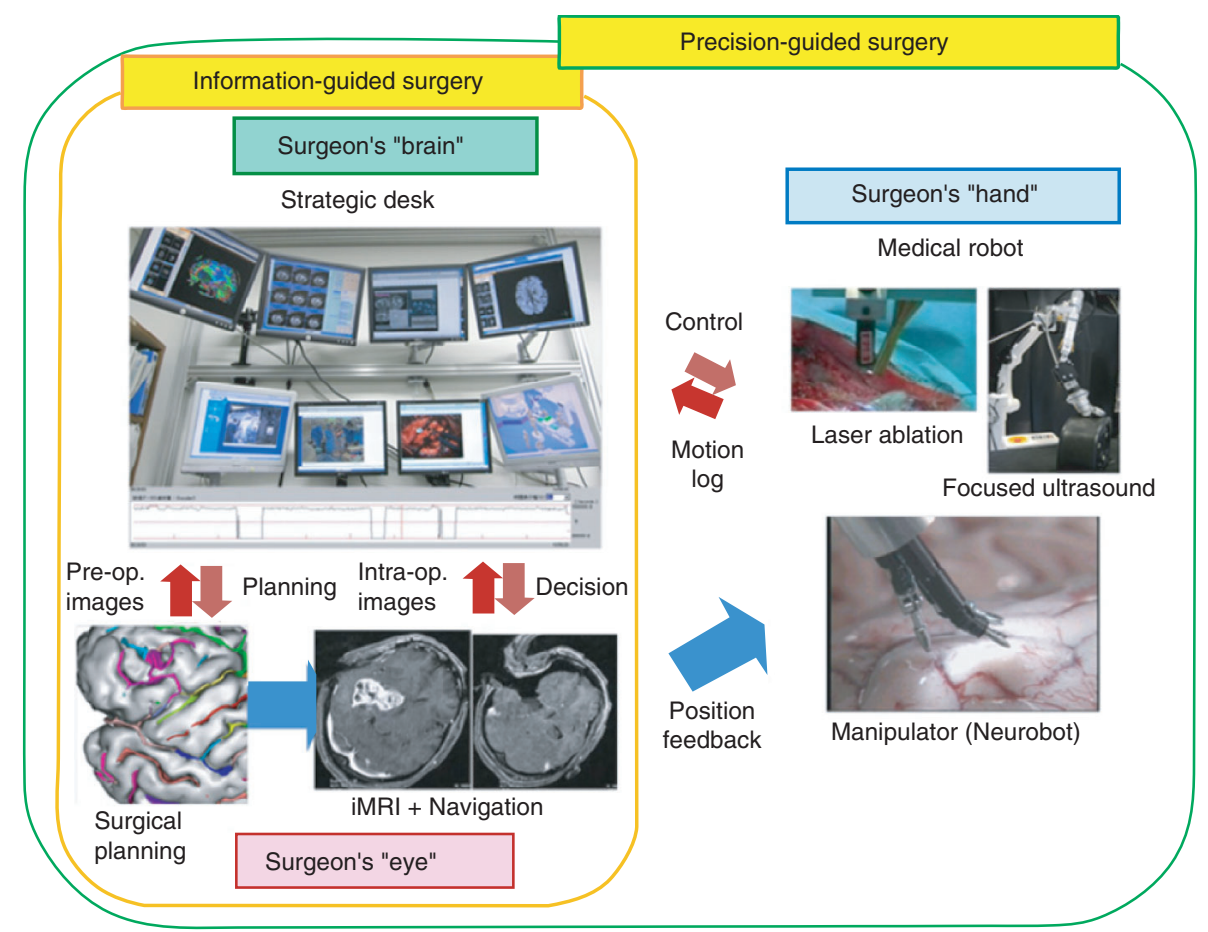

Figure 1. General framework of the information-guided and precision-guided brain surgery in Tokyo Women's Medical University. iMRI: intraoperative MRI. Pre-op.: preoperative. Intra-op.: intraoperative. 
the diagnostic and management data of the particular patient (advanced surgeon's "eye"), special computer-aided systems for comprehensive analyses of these data and simulation of the treatment course (advanced surgeon's "brain"), and dedicated instrumentarium for greater efficacy of the surgical manipulations (advanced surgeon's "hand").

The present report summarizes the experience with advanced information-guided surgical management of the intracranial gliomas gained in Tokyo Women's Medical University (TWMU) from 2000 to 2011. It describes the strategy for advanced intraoperative visualization of the neoplasm and identification of its borders with the adjacent brain tissue, highlights currently available computer-aided systems for facilitating and support of the surgical decision-making, shows the outcome of brain tumor surgery in the Intelligent Operating Theater (IOT), underlines clinical rationale for aggressive resection of gliomas, and presents some dedicated devices which have a significant potential to improve efficacy of the neurosurgical procedures in the nearest future while still in the preclinical stage of testing. It should be underlined that detailed analysis of the clinical results is beyond the scope of this study, which is rather directed on characterization of the advanced intraoperative technologies currently available for surgical management of glioma and presentation of our treatment concept.

\section{METHODS}

From March 2000 to August 2011, more than 1000 neurosurgical interventions for various intracranial pathology were performed in the IOT of TWMU. Approximately three quarters of these procedures, including 242 awake craniotomies, were directed on resection of intracranial gliomas. The present analysis of the advanced intraoperative technology and general surgical outcome did not require any special permission or approval by the committee for protection of human subjects in research.

\subsection{Advanced Intraoperative Technology of the IOT of TWMU}

\subsubsection{Intraoperative MRI}

The organization of the IOT of TWMU is illustrated in Figure 2. It is equipped with a low-field-strength (0.3 Tesla) open intraoperative magnetic resonance imaging (iMRI) scanner (AIRIS II ${ }^{\mathrm{TM}}$; Hitachi Medical Corporation, Tokyo, Japan) in a hamburger-like shape, with a $43 \mathrm{~cm}$ gantry gap and a disc-shaped permanent magnet producing vertical magnetic field with resonance frequency of $12.7 \mathrm{MHz}$. This scanner does not require a cooling system, thus significantly reducing its operating cost by approximately 10.000 Japanese yen (around US\$100) per month. The low magnetic field strength creates a narrow 5-gauss zone, extending 2 meters from both sides, 2.2 meters in front, 1.8 meters backwards, and 2.5 meters upwards, which allows for the surgeon to use some conventional surgical instruments (e.g., high-speed drill) in the working space outside such area. Nevertheless, all surgical devices used within the 5-gauss line, such as operating table (MOT2000-MRI; Mizuho Ltd., Tokyo, Japan) and operating microscope (MRI-30; Mitaka Co., Tokyo, Japan) are constructed from non-ferromagnetic material to prevent accidents and avoid image artifacts. The 


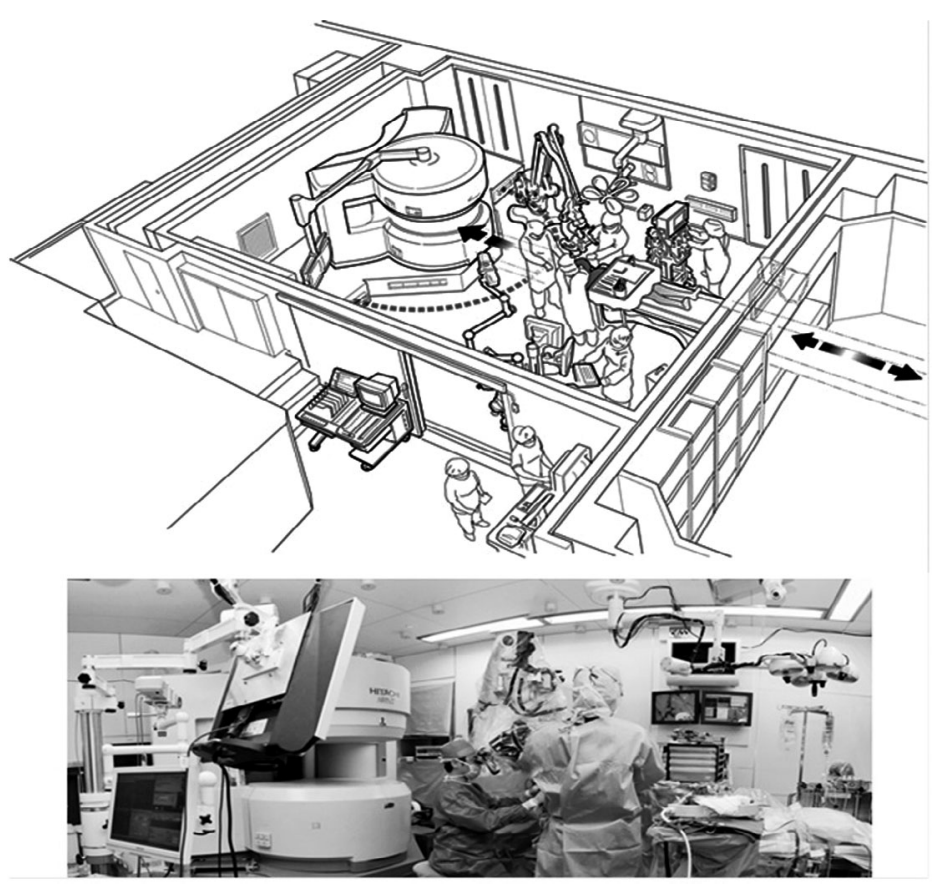

Figure 2. Schematic illustration and photograph of the intelligent operating theater in Tokyo Women's Medical University. Arrows indicate directions of the operating table movement during intraoperative MR investigations, dots correspond to 5-gauss line.

specially developed radiofrequency receiver coil integrated with the Sugita headholder (Head-holder coil; Mizuho Ltd., Tokyo, Japan) significantly improves the quality of the intraoperative images [1-5].

\subsubsection{Intraoperative Neuronavigation}

Simultaneous with installation of iMRI in IOT of TWMU, a special system for neuronavigation based on the intraoperative neuroimaging was developed in 2000 [1, 2]. Its main advantage is avoidance of the adverse effects caused by brain shift and deformation, allowing precise identification of the tumor position and its interrelationships with surrounding brain structures. To facilitate tumor removal and detection of the neoplastic remnants we use previously developed navigator for photon radiosurgery system (PRS navigator; Toshiba Medical Co. Ltd., Tokyo, Japan), which allows fast and easy updating of the information obtained with iMRI. The mislocalization error of the device is $0.8 \mathrm{~mm}$ in average, $1.5 \mathrm{~mm}$ at maximum, and $0.5 \mathrm{~mm}$ at minimum, and typically does not exceed $1 \mathrm{~mm}[1,2]$. The system permits co-registration, fusion and three-dimensional reconstruction of various images, and 
provides easy-to-understand information. The different areas of the perilesional brain can be color-coded according to the safety of manipulations and probable risk of complications. Additionally, the device may be integrated with special sound alarm automatically activated if surgical instruments are coming in close proximity to the high-risk area.

\subsubsection{Intraoperative Neurophysiological Monitoring and Brain Mapping with Awake} Craniotomy

Comprehensive intraoperative neurophysiological monitoring is routinely implemented during surgical procedures in the IOT of TWMU [5, 6]. The level of anesthesia, which may interfere with results of such examinations, is constantly monitored with bispectral index (BIS) monitor. Somatosensory evoked potentials (SEP) are recorded with stimulation of the median nerve. The values of the latencies and amplitudes obtained after craniotomy just before the dural incision are considered as an individual baseline. The central sulcus is localized based on the polarity inversion of the N20 component of SEP. Motor evoked potentials (MEP) are monitored using transcranial electrodes and measurement of the electromyograms at the thenar, quadriceps femoris, anterior tibial, and gastrocnemius muscles on both sides. Direct cortical mapping of the primary motor area is also performed.

\subsubsection{Neurochemical Navigation with 5-ALA and Intraoperative Histopathological Diagnosis}

During surgery for intracranial gliomas neurochemical navigation with 5-aminolevulinic acid (5-ALA) and intraoperative microscopic investigation of the pathological material on the frozen sections are routinely performed for identification of the remnants of the neoplasm and detailed histopathological characterization of the resected tissue [7].

\subsubsection{Intraoperative Examination Monitor for Awake Surgery (IEMAS)}

In 2004, a dedicated device called Intraoperative Examination Monitoring system for Awake Surgery (IEMAS) was developed by our group and incorporated into clinical practice during awake craniotomy for lesions located in or in the nearest vicinity to eloquent cortical areas [2, 5, 8]. As shown in Figure 3, this device integrates seven channels from video sources of various anatomical and functional parameters, including the view of the patient's face, examination task, level of sedation (BIS monitor), view of the surgical field, and display of the neuronavigation system. Surgeons can observe various intraoperative data simultaneously on one display and record it in the integrated format. All members of the surgical team can visualize and analyze the information practically without interruption of the surgical manipulations.

\subsubsection{Surgical Information Strategy Desk}

For appropriate analysis of various surgical data a special computer-assisted system, "Surgical Information Strategy Desk", was designed and installed in the surgical office [2, 5]. 
(A)

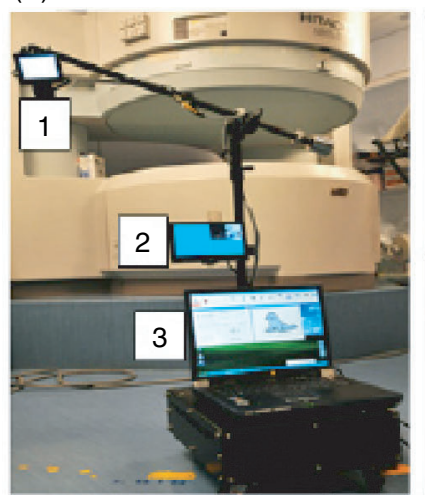

(B)

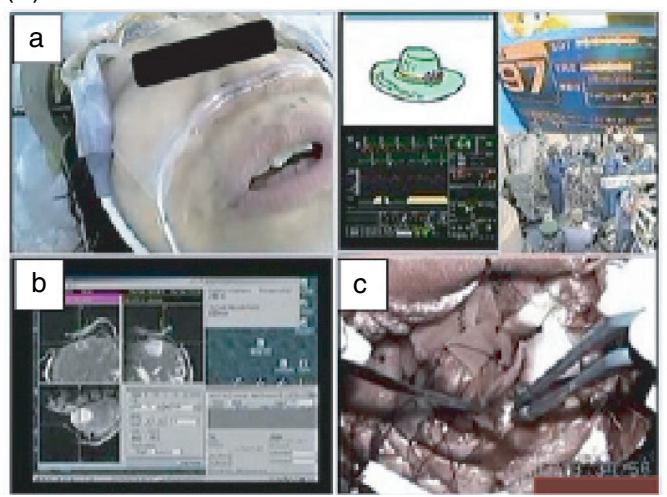

Figure 3. Integration of the multiple intraoperative data on the screen of IEMAS. (A) General view of the wireless modification of the device demonstrating its 3 main parts, patient's monitor (1), operator's monitor (2), and control box (3). (B) On display of the IEMAS, different intraoperative information can be integrated real time and shown in multiple windows: view of the patient's face and mimics (a) for checking the consciousness status and evaluation of response to test questions, anatomical data from the updated neuronavigation system (b) allowing localization of the exact position of the cortical stimulator, whereas view of the surgical field through the operative microscope (c) may be helpful for precise identification of the stimulation time.

All pre- and intra-operative information can be viewed through eight LCD displays. This system is located in a dedicated center for researchers specializing in medicine, biotechnology, pharmacology and engineering, as well as the basic sciences of chemistry and physics.

\subsection{Strategy for Management of Intracranial Glioma}

\subsubsection{Surgical Concept and Objectives}

The concept of the information-guided surgical management of gliomas based on the integration of various intraoperative anatomical, functional, and histopathological data is depicted in Figure 4.

The resection rate is mainly determined by 3 interrelated factors: tumor size, histopathological grade, and functional grade $[1,5,9]$. The goal of surgery is defined as maximal possible removal of the neoplasm, which can be radiologically total or subtotal removal, assessed by the size of the residual lesion within the functioning eloquent brain structures identified with neurophysiological monitoring and/or brain mapping. In general, the optimal result corresponds to elimination of the whole area of hyperintense signal detected on preoperative T2-weighted or FLAIR images. It can be often achieved in cases of low-grade neoplasms, but rarely attained in high-grade lesions surrounded by prominent 


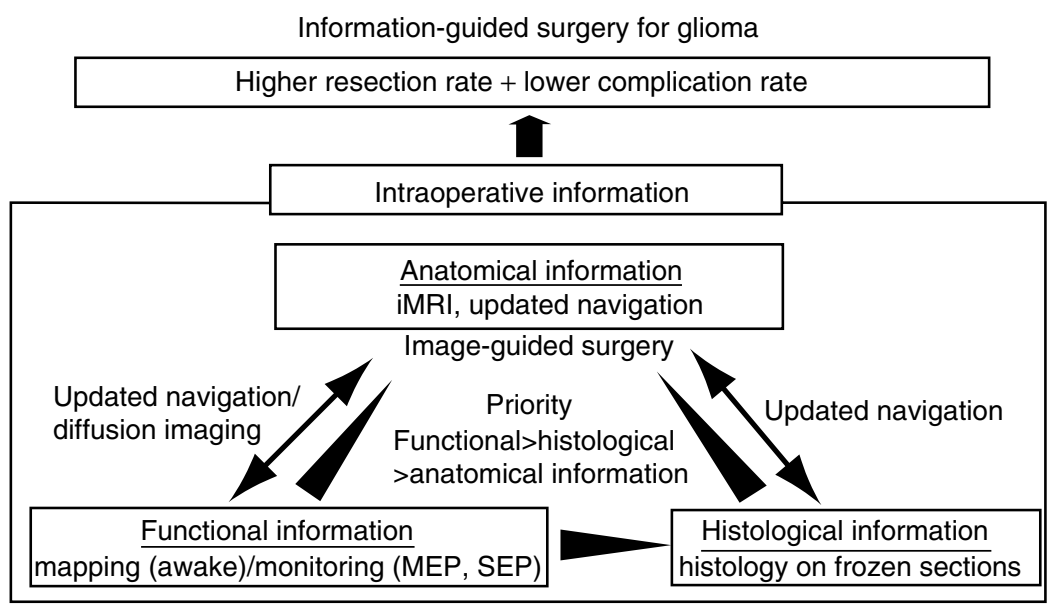

Figure 4. Main principle of the information-guided surgery for glioma. To maximize resection rate and minimize complication rate, anatomical, functional, histological, and metabolic data, etc. are needed. Anatomical data are integrated with functional and histological data by neuronavigation (section 2.1.2); however, functional data have definite priority in surgical decision-making. iMRI: intraoperative MRI. MEP: motor evoked potentials. SEP: somatosensory evoked potentials.

peritumoral edema. In the latter case, the surgery is usually aimed at maximal removal of the contrast-enhanced area. Finally, in the rare occasions of extensive tumors affecting functioning eloquent brain structures the surgery is aimed at removal of the most metabolically active part of the neoplasm detected by ${ }^{11} \mathrm{C}$-methionine uptake on PET.

\subsubsection{Guidance of Tumor Resection}

The initial iMRI investigation with subsequent installation of data in the neuronavigation system is performed after craniotomy and completion of the approach to the tumor. After removal of the neoplasm, the investigation is repeated for assessment of the completeness of resection, identification of the residual lesion or possible adverse effects such as hemorrhage. If residual glioma is identified and considered suitable for additional resection, the newly obtained iMRI data are installed in the neuronavigation device and further removal of the neoplasm is performed using this updated information. After completion of such additional resection iMRI is repeated once again. However, such anatomical data are used for removal of the bulk of the tumor only, whereas resection of the residual neoplasm is based not only on the anatomical images, but on the results of neurochemical navigation with 5-ALA and histopathological investigation of the walls of the surgical cavity [9].

It should be noted that neither anatomical data obtained with iMRI nor histopathological information on the presence of residual neoplasm is sufficient for 
guidance of the tumor resection, especially if the lesion is located near or within functionally-important cerebral structures. In all circumstances, the first priority in surgical decision-making is given to functional information provided by intraoperative brain mapping and comprehensive neurophysiological monitoring.

\subsubsection{Evaluation of Resection Rate}

Resection rate of intracranial gliomas is evaluated by postoperative 1.5 Tesla MRI. In cases of contrast-enhanced tumors the investigation is usually performed within 48 to 72 hours after surgery, while after removal of non-enhancing low-grade neoplasms it is frequently delayed up to 5 to 7 days for the resolution of possible postoperative brain edema which may result in overestimation of the residual lesion [10]. If elimination of 95\% or more of the mass in terms of signal hyperintensity on T2-weighted images in cases of low-grade neoplasms, or contrast-enhanced area on postgadolinium T1weighted images in cases of high-grade ones is confirmed, the resection is considered total. Such cut-off level corresponds to the grading of resection of gliomas adopted by The Committee of Brain Tumor Registry of Japan [11]. Volumetric methods for postoperative evaluation of brain tumors are not employed routinely, since they are not required by the Response Assessment in Neuro-Oncology (RANO) working group and are still considered a field of major research [12,13].

\subsubsection{Postoperative Management of Brain Tumors}

After the surgery for intracranial gliomas, all patients receive regular clinical and radiological examinations by the treating neurosurgeons in the outpatient clinic. Our strategy for postoperative management of such tumors is presented in Table 1.

Table 1. Postoperative management of newly diagnosed intracranial gliomas

\begin{tabular}{|c|c|c|}
\hline \multirow{2}{*}{$\begin{array}{l}\text { WHO histopathological } \\
\text { tumor grade }\end{array}$} & \multicolumn{2}{|c|}{ Degree of surgical resection } \\
\hline & Total (95\% and more) & Incomplete/Biopsy \\
\hline$\underline{I}$ & Observation & Observation \\
\hline $\begin{array}{l}\text { II (oligodendroglioma, } \\
\text { oligoastrocytoma) IDHI } \\
\text { mutation (+) and/or } \\
\text { LOH } 1 \mathrm{p} / 19 \mathrm{q}(+)\end{array}$ & Observation & Chemotherapy with nidran \\
\hline II (any other) & Observation & $\begin{array}{l}\text { Fractionated Radiotherapy } \\
\text { (total dose } 50 \text { Gy) plus } \\
\text { concomitant and adjuvant } \\
\text { chemotherapy with nidran }\end{array}$ \\
\hline III & \multicolumn{2}{|c|}{$\begin{array}{l}\text { Fractionated Radiotherapy (total dose } 60 \mathrm{~Gy} \text { ) plus } \\
\text { concomitant and adjuvant chemotherapy with nidran }\end{array}$} \\
\hline IV & \multicolumn{2}{|c|}{$\begin{array}{l}\text { Fractionated Radiotherapy (total dose } 60 \mathrm{~Gy} \text { ) plus } \\
\text { concomitant and adjuvant chemotherapy with temozolomide }\end{array}$} \\
\hline
\end{tabular}


The diagnosis of tumor re-growth or recurrence is based on the joint opinion of the neurosurgeon and neuroradiologist, and is defined as $25 \%$ or more increase of the volume of the previously existent lesion or appearance of the new lesion on postcontrast $\mathrm{T}_{1}$-weighted $\mathrm{MR}$ images. At the time of tumor progression the vast majority of patients receive salvage treatment, which usually include re-resection of the neoplasm, stereotactic radiosurgery, chemotherapy, vaccine therapy, or their various combinations.

\subsubsection{Collection and Assessment of Clinical Information}

All pre-operative, intraoperative, and follow-up clinical data are collected by dedicated staff members not involved in the direct treatment of patients. This information is preserved in constantly maintained computer database and available for immediate use upon the request of attending neurosurgeons.

\section{RESULTS}

The length of brain tumor surgery in IOT of TWMU usually varied from 8 to 12 hours depending on the size and location of the neoplasm, and the number of iMRI investigations.

Radiologically total removal of glioma was achieved in $46 \%$ of cases. Subtotal removal was performed in $53 \%$ of patients, leaving the remnants of the neoplasm within the functioning eloquent brain structures identified by intraoperative brain mapping and comprehensive neurophysiological monitoring. The mean resection rate was $92 \%$ for all histopathological grades, with the medians of 91\%, 93\%, and $92 \%$ for WHO grade II, III, and IV neoplasms, respectively. The median residual tumor volume was $0.17 \mathrm{cc}$. In general, the residual tumor volume was greater in neoplasms of higher histopathological and functional grade, but such trend did not reach statistical significance. Unexpected residual tumor was never disclosed by postoperative MRI (1.5 Tesla) which has a much higher magnetic field strength compared to iMRI (0.3 Tesla).

Improvements of pre-existing signs and symptoms were noted in $13 \%$ of the patients. Permanent neurological deterioration after surgery occurred in $12 \%$ of cases, but it reduced to $9 \%$ after intentional decrease of the resection rate in our latest cohort of patients with WHO grade II and III gliomas. Wound infection after removal of the tumor was observed in $2 \%$ of cases. Postoperative hemorrhage never occurred in any case.

In patients with available long-term follow-up the prognosis was significantly worse for cases of WHO grade IV gliomas, but did not reach statistically significant differences between WHO grades II and III tumors [14], as illustrated in Figure 5. Among individuals with WHO grades II, III, and IV gliomas, who were followed for more than 2 years after surgery, the corresponding actuarial 5-year survival rates were $87 \%, 69 \%$, and $19 \%$, respectively. In patients with low-grade glioma the extent of tumor resection was significantly associated with prolonged overall survival, progression-free survival, and malignant-progression-free survival. 


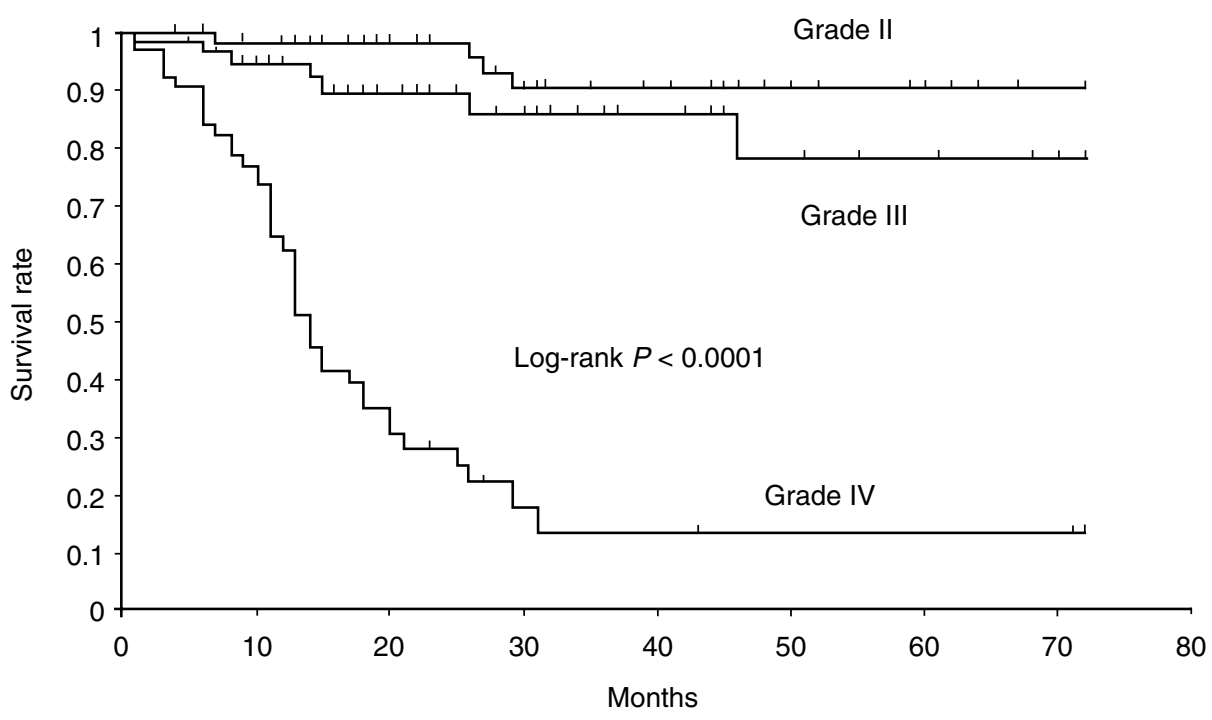

Figure 5. Overall survival rate of patients with gliomas of different histopathological grades. Based on the Cox proportional hazard model, there is no significant difference between neoplasms of grades II and III ( $p=0.1742$ ), but there is a significant difference between grades III and IV ( $\mathrm{p}<0.0001)$. Source: Shinohara et al. [14], adopted with permission.

\section{DISCUSSION}

The well-known benefits of resective surgery in cases of glioma include relief of compression of the tumor bulk on the surrounding brain (important for neurological improvement) and establishment of the precise histopathological diagnosis, which determines choice of the adjuvant therapy, optimal follow-up, and predicts prognosis. Additionally, reduction of the mass volume can render postoperative radiochemotherapy more effective [15].

Extensive removal of the tumor may positively influence patients' survival [16]. Stummer et al. [17] adjusted biases of age and eloquent area location in the dataset of a randomized study on use of neurochemical navigation with 5-ALA during resection of glioblastoma multiforme (GBM), and found that median overall survival time after complete removal of the contrast-enhanced lesion was significantly longer compared to incomplete resection (17 months vs. 12 months). Further, in the report on a randomized trial of combined chemotherapy for anaplastic gliomas performed by the European Organization for Research and Treatment of Cancer (EORTC), the overall survival was better after complete tumor removal compared to partial removal or biopsy [18]. It was demonstrated that $98 \%$ or more resection of GBM is associated with significant improvement of the long-term outcome [19], and the same trend was recently revealed even at $78 \%$ resection rate [20]. Similar results were reported by the Committee of Brain Tumor Registry of Japan [11] that analysis 
of 9,234 cases of malignant gliomas showed that more than $95 \%$ of tumor removal is associated with survival advantage, while such resection rate was attained in one third of cases only. Of special importance is the fact that aggressive resection of the neoplasm may provide survival advantage even in patients with prognostically unfavorable RPA classes IV and V [21].

As reported above in the RESULTS section, the 5-year survival rates of our patients were $87 \%, 69 \%$, and $19 \%$ for WHO grades II, III, and IV gliomas, respectively, compared to $41 \%$ for all gliomas, $22 \%$ for high-grade ones, and $7 \%$ for glioblastomas in general neurosurgical practices, according to the Committee of Brain Tumor Registry of Japan [11]. It should be specifically emphasized that complete surgical resection is highly desirable, but not the ultimate goal of surgery for glioma; therefore it should not be performed if it may cause major permanent postoperative neurological morbidity or lead to significant decline of the quality of life.

\subsection{Advanced Surgeon's "Eye"}

4.1.1. Intraoperative MRI

The iMRI systems were first introduced to neurosurgery at the end of 1990's. Since then, such devices have been used for real-time imaging for surgical manipulations, for assessment of the extent of tumor resection, and evaluation of the intraoperative complications. While real-time guidance of the surgical manipulations with iMRI is theoretically presumed to be the most effective, such systems usually reduce the work space available and necessitate all surgical devices to be made of nonferromagnetic materials. In contrast, if iMRI scanning is performed at some temporary break points during surgical procedure, it can provide a higher degree of freedom for the surgeon and allow the use of standard (not MRI-compatible) neurosurgical instrumentarium [1, 2, 5, 22-24]. It should be noted that employment of any type of iMRI system increases the operation time, because MR imaging itself is a time-consuming process.

During the last decade there is a trend of employing iMRI scanners with high magnetic field strength of 1.5 and 3 Tesla [25-27]. Their advantages include high image quality, possibility to attain diffusion tensor and spectroscopic images, as well as short scanning time [25]. Increase of magnetic field strength, however, is associated with greater possibility of image distortion artifacts. The risk of the latter is 5 times greater in a 1.5 Tesla MR scanner compared to a 0.2 Tesla one [28]. Additionally, the maintenance cost of high-field-strength iMRI scanners is significantly higher.

The magnetic field strength of the iMRI installed in IOT of TWMU is only 0.3 Tesla; nevertheless, it is able to obtain not only volumetric MR images, but to perform MR angiography and functional investigations $[2,5,22]$ by using a specially developed Head-holder coil. Integration of such coil with modified Komai stereotactic frame allows stereotactically guided surgical procedures under the control of iMRI [2]. The most important achievement is the ability to obtain intraoperative diffusion-weighted images (iDWI) which provide nearly real-time information on spatial interrelationships between the pyramidal tract, the lesion, and positions of the surgical instruments 
including the electrical stimulator for subcortical functional mapping [3, 4]. iDWI can effectively prevent inadvertent injury of the pyramidal tract and thus avoid postoperative deterioration of the motor function. The estimated positional accuracy of iDWI in our practice is within $5 \mathrm{~mm}$, comparable to the conducting depth of the electrical stimulation for subcortical brain mapping [6]. It should be noted, however, that diffusion anisotropy of the white matter tracts may be reduced due to presence of the tumor or peritumoral edema, and possibly leading to poor visualization of the pyramidal tract on the affected side.

Recent prospective randomized trial of Senft et al. [29] demonstrated that iMRI has significant positive impact on the resection rate. In $96 \%$ of cases it allowed complete removal of the contrast-enhancing intracranial gliomas amenable for aggressive surgery, whereas in one third of patients use of intraoperative imaging directly led to extended tumor resection. The volume of the residual neoplasm after iMRI-guided surgery was significantly smaller than after conventional procedure, and fewer patients in the former group had progressive disease 6 months after intervention. Overall, the results were considered comparable to previously reported efficacy of the intraoperative neurochemical navigation with 5-ALA, although the two methods were not directly compared [29]. In our practice both techniques are constantly applied for optimal identification of the residual neoplasm and evaluation of its interrelationships with the functioning brain tissue.

\subsubsection{Neurochemical Navigation with 5-ALA}

Neurochemical navigation with 5-ALA during resection of gliomas is based on the effect of its accumulation in the tumor cells and areas with impaired blood-brain barrier (BBB) and further conversion into protoporphyrin IX whose fluorescence is visible under ultraviolet light. This method can be effectively used for identification of the neoplastic tissue and its differentiation from the brain itself, which may be particularly important at the periphery of the neoplasm. Large prospective multicenter randomized phase III trial clearly demonstrated that compared to conventional microsurgery use of neurochemical navigation with 5-ALA results in significantly greater proportion of complete resections of glioblastoma (defined as absence of the contrast-enhancing area on postoperative MRI), smaller volume of the residual tumor, and better progressionfree survival 6 months after intervention [30].

It should be noted, however, that erroneous results of neurochemical navigation with 5-ALA are possible. False negative results may occur in low-grade gliomas due to relative preservation of the $\mathrm{BBB}$ or the presence of the adherent blood on the tissue surface since erythrocytes can absorb ultraviolet light, which in such case does not reach protoporphyrin IX in the pathological tissue [7]. Contrarily, false positive fluorescence may be observed in the areas with high vascularization, reactive astrocytosis, or BBB impairment in the absence of the neoplastic elements [7]. Regular intraoperative histopathological investigation of the resected tissue on the frozen sections effectively complement neurochemical navigation and may be efficiently used for guidance of the tumor resection, identification of the neoplastic remnants, and confirmation of completeness of the lesion removal. 


\subsection{Advanced Surgeon's "Brain"}

4.2.1. Importance of the Neurophysiological Information during Glioma Resection

It should be clear that anatomical and histopathological data alone, even if obtained with iMRI, neurochemical navigation with 5-ALA, and regular intraoperative histopathological investigations of the walls of the surgical cavity, are not sufficient for guidance of the glioma resection. Location of the eloquent brain structures has known individual variability and may be displaced during growth of the neoplasm, thus necessitating comprehensive neurophysiological monitoring and intraoperative cortical and subcortical brain mapping with or without awake craniotomy, and further integration of the neurophysiological, histopathological, and anatomical data within the updated neuronavigation system [6, 31-34]. In all circumstances, the first priority in surgical decision-making should be given to functional information, especially in cases with critical location of the neoplasm [5, 9, 33, 34].

\subsubsection{Intraoperative Examination Monitor for Awake Surgery (IEMAS)}

The effectiveness of the information-guided tumor resection is strongly dependent on the quality of the available information. Particularly, during surgery for cerebral glioma preservation of the cortical speech centers is of paramount importance for preventing postoperative aphasia. It enforces awake craniotomy with intraoperative brain mapping for localization of the functionally important structures [35-37]. Such technique requires synchronization of the cortical electrical stimulation and functional testing for precise identification of the targeted region. It usually takes two members of the surgical team to perform the task, with one conducting the examination test to the patient and the other applying cortical electrical stimulation. To simplify this process, we developed the dedicated IEMAS which integrates various types of related information on one screen. The device is currently in the process of commercialization and receiving governmental authorization. Its latest improvement incorporated wireless audio-visual transmitting technology with paired signal transmitters and receivers for transfer of video images and verbal information [8].

\subsubsection{Surgical Information Strategy Desk}

A variety of data are involved in a brain tumor removal surgery. "Surgical Information Strategy Desk" was developed to integrate different information and facilitate surgical decision-making $[2,5]$. It provides an opportunity for the senior surgeon to observe simultaneously preoperative high-resolution diagnostic images by high-field MR scanner, surgical planning through computer simulation, surgical procedures performed by junior colleagues, intraoperative MR images, real-time update neuronavigation data, neurophysiological monitoring, histopathological investigation, etc. Using these data, optimal solutions for the ever changing surgical scenario can be effectively reached and communicated with the surgical staff. Various types of information are transferred to the command center, and distributed among other specialists, such as pathologist and engineer, via closed intra-hospital network. This enables emergency consulting service

even if the specialists are located far away from the operating room. When surgical staff requests consultation, they just have to access the network, where all surgical data are 
progressively collected and integrated via intra-hospital network from their offices. Theoretically, monitoring the surgical procedure by a senior surgeon from a distant site away from the operating theater may create problems in case of some troubles, and may even have medico-legal implications. However, we never had such negative experience therefore its risk should not be overestimated.

Various intraoperative data incorporated into "Surgical Information Strategy Desk" are used not only during surgical procedure, but stored on the hard disk for further analysis. Such information may be used later for evaluation of the surgical procedure and analysis of the outcome, for simulation and creation of the surgical plan for future cases, and for educational purposes in training junior neurosurgeons. Another direction of use of the intraoperative data, which is currently implemented in our laboratory, is surgical workflow analysis aiming at simplification and optimization of the surgical procedures.

\subsubsection{Analysis of Staff Movements inside the Operating Theater}

Currently we are trying to establish the links between events on the recorded video images of the surgical procedure or of the operating theater and details of surgery particularly the adverse and undesirable events [38]. We hypothesize that when accident occurs, the movements of the surgical staff should increase, which should be reflected on the video file's size. In the interframe video compression algorithm, the subtraction data between two continuous frames is the dominant factor for determination of the video file size. If the surgical staff members increase their movements, the subtraction data increase and consequently the file size increases. Therefore, such method can be effectively employed to measure the quantity of movements on the video. To achieve our objective, the recorded video image is separated into a series of one-minute files. The size of each file corresponds to the number of movements within a specific period of time. It is therefore easy to identify occurrences of accidents during the surgery. In addition to nuisances, this method can further identify different events happening in the operating room. It was found, that the size of the video file well correspond to the stage of the surgical procedure, such as skull drilling, microsurgical tumor removal, intraoperative MR scanning. At present we are applying this method for automatic recognition of the surgical stage. In the meantime, statistical analysis of the video recordings from multiple surgical procedures may establish the "normal" or "standard" amount of the surgical staff motions during each stage of procedure, which opens a way to indirect identification of the various intraoperative accidents.

\subsection{Advanced Surgeon's "Hand"}

\subsubsection{Robotic Neurosurgery}

Incorporation of robotic systems into neurosurgical practice can potentially increase the precision of surgical manipulations, and significantly reduce the risk of neurological deficits if surgery is performed in highly vulnerable brain areas. The robotic systems can perform automatic distinction between the tumor and surrounding tissue, and may provide an opportunity for highly selective management of the neoplasm with extremely high precision [39]. Other potential advantages of robotics include the opportunity to perform manipulations in extremely limited space, and the possibility of initial computer-aided 
modeling and simulation of the planned surgical action. The introduction of the robotic technologies into clinical medicine started in the 1990s with such system as da Vinci ${ }^{\mathrm{TM}}$ (Intuitive Surgical Inc., Sunnyvale, CA, U.S.A). In the meantime, we developed a special neurosurgical robotic system, designated as the Hyper Utility Mechatronic Assistant for Neurosurgery (HUMAN) or NeuRobot (commercialized by Hitachi Medical Corporation, Tokyo, Japan). For the first time in the world it was clinically implemented at Shinshu University (Matsumoto, Japan) in August 2002 and proved the possible practical utility, particularly for the third vetnriculostomy [40]. Currently, an advanced iMRI-compatible model of this device is undergoing experimental testing.

The next generation of neurosurgical robots features integration of advanced intraoperative neurovisualization and computer technology for comprehensive data assessment, and will be able to perform highly selective automatic removal of the neoplasm with preservation of adjacent normal tissue (precision-guided surgery). We have been developing a manipulator based on the integration of the chemical neuronavigation for localizing the neoplasm in the surgical field and micro-laser for its ablation as illustrated on Figure 6. Specially designed camera incorporated in this
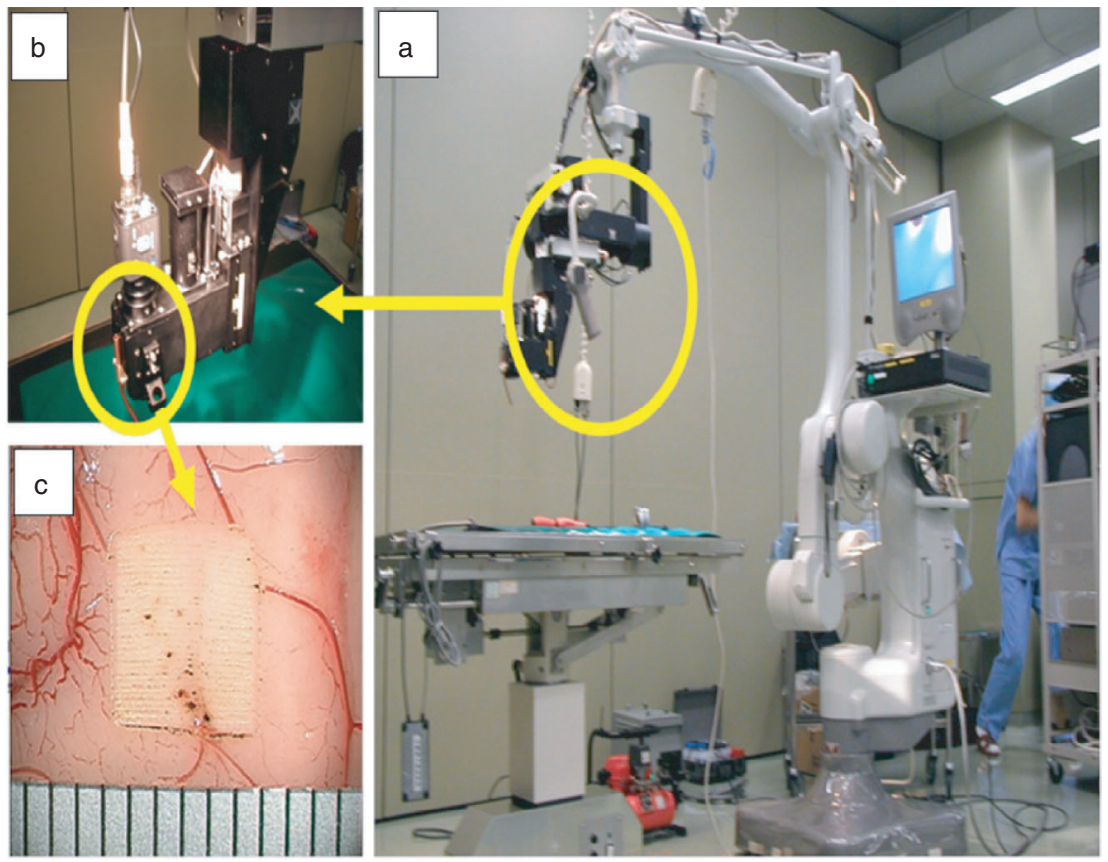

Figure 6. Neurosurgical robotic system for tumor ablation. The device is fixed on the head of the operative microscope (a), and includes a micro-laser and a specially designed camera (b) for automatic determination of the management area to allow extremely limited thermal action with better than $0.5 \mathrm{~mm}$ positioning accuracy $(\mathrm{c})$. 
device allows automatic determination of the management area. A mid-infrared continuous wave micro-laser with a wave length of 2.8 micrometers provides optimal absorption parameters of the brain tissue and extremely limited thermal action. The system has a better than $0.5 \mathrm{~mm}$ positioning accuracy. All surgical actions are planned and controlled by the neurosurgeon at a distant desk. It should be noted that this device has been developed not for removal of the whole bulk of the tumor, but for management of its residuals in the highly eloquent brain structures where high levels of preciseness and safety are needed. It is expected that such highly selective pin point neurosurgery will enable removal of the neoplasm while keeping functionally important brain structures intact. The clinical testing of the device is expected in the nearest future.

\subsubsection{High Intensity Focused Ultrasound (HIFU)}

Another development among next generation of the therapeutic systems for brain tumor management is the High Intensity Focused Ultrasound (HIFU) developed as a prototype for novel high-precision device for guiding surgical procedures (joint project of TWMU, Tohoku University and Hitachi Medical Corporation). Its performance parameters, targeting accuracy, and therapeutic efficacy are currently under evaluation in the Intelligent Animal Operating Theater in Tokyo Women's Medical UniversityWaseda University Joint Institution (TWIns) for Advanced Biomedical Sciences, where the device is incorporated with the ultrasound imaging and iMRI systems. A set of animal experiments have been conducted to achieve from $75 \%$ to $80 \%$ reduction of the tumor growth and establishment of the optimal technical parameters and safety regulations for further application of the device to clinical trials.

\section{CONCLUSION}

Information-guided surgery of intracranial gliomas based on intraoperative integration of various anatomical, neurophysiological, and histopathological data enables aggressive tumor resection with minimal risk of permanent postoperative neurological morbidity, and may result in significant prolongation of patients' survival. Continual progress in advanced visualization, comprehensive medical information management, and robotics bring forth a great potential to further increase the efficacy and safety of the surgical procedures.

\section{ACKNOWLEDGEMENTS}

The authors wish to express their gratitude to the members and technical staff of the Departments of Neurosurgery and Anesthesiology, Surgical Facility Unit, and Faculty of Advanced Techno-Surgery of the Tokyo Women's Medical University, as well as to maintenance team of the Hitachi Medical Corporation, for the long-term friendly and fruitful collaboration during clinical work both inside and outside the Intelligent Operating Theater, which allows us to provide the best possible care for the patients with brain tumors. Special thanks are devoted to Prof. Yoshikazu Okada, Prof. Tomokatsu Hori, and Dr. Masahiko Tanaka for their constant support of our clinical and research activities.

This study is partly supported by The Japan Society for the Promotion of Science (JSPS) through the "Funding Program for World-Leading Innovative R\&D on Science 
and Technology (FIRST Program)" initiated by the Council for Science and Technology Policy (CSTP), The Global COE Program "Multidisciplinary Education and Research Center for Regenerative Medicine (MERCREM)" from the Ministry of Education, Culture, Sports Science, and Technology of Japan, Health and Labor Sciences Research Grants provided by the Ministry of Health, Labor and Welfare of Japan, Core Research of Evolutional Science and Technology (CREST) grant from the Japan Science and Technology Agency, as well as NEDO P10003 "Intelligent Surgical Instruments Project", KAKENHI (22650115, 10F00128, 20791018, 22300093), and support from the Ministry of Economy, Trade and Industry (METI) of Japan.

\section{CONFLICT OF INTEREST}

The authors indicated no potential conflicts of interest.

\section{REFERENCES}

[1] Muragaki Y., Iseki H., Maruyama T., Kawamata T., Yamane F., Nakamura R., Kubo O., Takakura K., Hori T. Usefulness of intraoperative magnetic resonance imaging for glioma surgery. Acta Neurochirurgica Supplement. 2006, 98, 67-75.

[2] Iseki H., Nakamura R., Muragaki Y., Suzuki T., Chernov M., Hori T., Takakura K. Advanced computer-aided intraoperative technologies for information-guided surgical management of gliomas: Tokyo Women's Medical University experience. Minimally Invasive Neurosurgery. 2008, 51(5), 285-291.

[3] Ozawa N., Muragaki Y., Nakamura R., Iseki H. Intraoperative diffusion-weighted imaging for visualization of the pyramidal tracts. Part I: pre-clinical validation of the scanning protocol. Minimally Invasive Neurosurgery. 2008, 51(2), 63-66.

[4] Ozawa N., Muragaki Y., Nakamura R., Iseki H. Intraoperative diffusion-weighted imaging for visualization of the pyramidal tracts. Part II: clinical study of usefulness and efficacy. Minimally Invasive Neurosurgery. 2008, 51(2), 67-71.

[5] Muragaki Y., Iseki H., Maruyama T., Chernov M., Suzuki T., Yoshimitsu K., Tamura M., Ikuta S., Nitta M., Saito T., Okamoto J., Takakura K. Advanced surgical management of gliomas: technological requirements, concept of information-guided resection, and clinical results, in: Abujambra A.L. (ed.) Diagnostic Techniques and Surgical Management of Brain Tumors, InTech, Croatia, 2011, 517-534.

[6] Ozawa N., Muragaki Y., Nakamura R., Iseki H. Identification of the pyramidal tract by neuronavigation based on intraoperative diffusion-weighted imaging combined with subcortical stimulation. Stereotactic and Functional Neurosurgery. 2009, 87(1), 18-24.

[7] Ando T., Kobayashi E., Liao H., Maruyama T., Muragaki Y., Iseki H., Kubo O., Sakuma I. Precise comparison of protoporphyrin IX fluorescence spectra with pathological results for brain tumor tissue identification. Brain Tumor Pathology. 2011, 28(1), 43-51.

[8] Yoshimitsu K., Maruyama T., Muragaki Y., Suzuki T., Saito T., Nitta M., Tanaka M., Chernov M., Tamura M., Ikuta S., Okamoto J., Okada Y., Iseki H. Wireless modification of the intraoperative examination monitor for awake surgery. Neurologia Medico-Chirurgica. 2011, 51(6), 472-476.

[9] Muragaki Y., Iseki H., Maruyama T., Tanaka M., Shinohara C., Suzuki T., Yoshimitsu K., Ikuta S., Hayashi M., Chernov M., Hori T., Okada Y., Takakura K. Information-guided surgical management of gliomas using low-field-strength intraoperative MRI. Acta Neurochirurgica Supplement. 2011, 109, $67-72$.

[10] Belhawi S.M.K., Hoefnagels F.W.A., Baaijen J.C., Aliaga E.S., Reijneveld J.C., Heimans J.J., Barkhof F., Vandertop W.P., De Witt Hamer P.C. Early postoperative MRI overestimates residual tumour after resection of gliomas with no or minimal enhancement. European Radiology. 2011, 21(7), 1526-1534. 
[11] Report of brain tumor registry of Japan (1984-2000) $12^{\text {th }}$ edition. Neurologia Medico-Chirurgica. 2009, 49 (Suppl.), 1-101.

[12] Lutz K., Radbruch A., Wiestler B., Baumer P., Wick W., Bendszus M. Neuroradiological response criteria for high-grade gliomas. Clinical Neuroradiology. 2011, 21(4), 199-205.

[13] Vogelbaum M.A., Jost S., Aghi M.K., Heimberger A.B., Sampson J.H., Wen P.Y., Macdonald D.R., Van den Bent M.J., Chang S.M. Application of novel response/progression measures for surgically delivered therapies for gliomas: Response Assessment in Neuro-Oncology (RANO) working group. Neurosurgery. 2012, 70(1), 234-244.

[14] Shinohara C., Muragaki Y., Maruyama T., Shimizu S., Tanaka M., Kubota Y., Oikawa M., Nakamura R., Iseki H., Kubo O., Takakura K., Hori T. Long-term prognostic assessment of 185 newly diagnosed gliomas: Grade III glioma showed prognosis comparable to that of Grade II glioma. Japanese Journal of Clinical Oncology. 2008, 38(11), 730-733.

[15] Stummer W., van den Bent M.J., Westphal M. Cytoreductive surgery of glioblastoma as the key to successful adjuvant therapies: new arguments in an old discussion. Acta Neurochirurgica. 2011, 153(6), 1211-1218.

[16] Proescholdt M.A., Macher C., Woertgen C., Brawanski A. Level of evidence in the literature concerning brain tumor resection. Clinical Neurology and Neurosurgery. 2005, 107(2), 95-98.

[17] Stummer W., Reulen H.J., Meinel T., Pichlmeier U., Schumacher W., Tonn J.C., Rohde V., Oppel F., Turowski B., Woiciechowsky C., Franz K., Pietsch T. Extent of resection and survival in glioblastoma multiforme: identification of and adjustment for bias. Neurosurgery, 2008, 62(3), 564-576.

[18] van den Bent M.J., Carpentier A.F., Brandes A.A., Sanson M., Taphoorn M.J., Bernsen H.J., Frenay M., Tijssen C.C., Grisold W., Sipos L, Haaxma-Reiche H., Kros J.M., van Kouwenhoven M.C., Vecht C.J., Allqeier A., Lacombe D., Gorlia T. Adjuvant procarbasine, lomustine, and vincristine improves progression-free survival but not overall survival in newly diagnosed anaplastic oligodendrogliomas and oligoastrocytomas: a randomized European Organisation for Research and Treatment of Cancer phase III trial. Journal of Clinical Oncology. 2006, 24(18), 2715-2722.

[19] Lacroix M., Abi-Said D., Fourney D.R., Gokaslan Z.L., Shi W., DeMonte F., Lang F.F., McCutcheon I.E., Hassenbusch S.J., Holland E., Hess K., Michael C., Miller D., Sawaya R. A multivariate analysis of 416 patients with glioblastoma multiforme: prognosis, extent of resection, and survival. Journal of Neurosurgery. 2001, 95(2), 190-198.

[20] Sanai N., Polley M.Y., McDermott M.W., Parsa A.T., Berger M.S. An extent of resection threshold for newly diagnosed glioblastomas. Journal of Neurosurgery. 2011, 115(1), 3-8.

[21] Pichlmeier U., Bink A., Schackert G., Stummer W., and the ALA Glioma Study Group. Resection and survival in glioblastoma multiforme: an RTOG recursive partitioning analysis of ALA study patients. Neuro-Oncology. 2008, 10(6), 1025-1034.

[22] Gasser T., Szelenyi A., Senft C., Muragaki Y., Sandalcioglu I.E., Sure U., Nimsky C., Seifert V. Intraoperative MRI and functional mapping. Acta Neurochirurgica Supplement. 2011, 109, 61-65.

[23] Hadani M., Spiegelman R., Feldman Z., Berkenstadt H., Ram Z. Novel, compact, intraoperative magnetic resonance imaging-guided system for conventional neurosurgical operating rooms. Neurosurgery. 2001, 48(4), 799-809.

[24] Senft C., Franz K., Ulrich C.T., Bink A., Szelenyi A., Gasser T., Seifert V. Low field intraoperative MRI-guided surgery of gliomas: a single center experience. Clinical Neurology and Neurosurgery. 2010, 112(3), 237-243.

[25] Hall W.A., Truwit C.L. Intraoperative magnetic resonance imaging. Acta Neurochirurgica Supplement. 2011, 109, 119-129.

[26] Nimsky C., Ganslandt O., Von Keller B., Romstock J., Fahlbusch R. Intraoperative high-field-strength MR imaging: implementation and experience in 200 patients. Radiology. 2004, 233(1), 67-78.

[27] Pamir M.N., Ozduman K., Dincer A., Yildiz E., Peker S., Ozek M.M. First intraoperative, shared resource, ultrahigh-field 3-Tesla magnetic resonance imaging system and its application in low-grade glioma resection. Journal of Neurosurgery. 2010, 112(1), 57-69. 
[28] Fransson A., Andreo P., Potter R. Aspects of MR image distortions in radiotherapy treatment planning. Strahlentherapie und Onkologie. 2001, 177(2), 59-73.

[29] Senft C., Bink A., Franz K., Vatter H., Gasser T., Seifert V. Intraoperative MRI guidance and extent of resection in glioma surgery: a randomized, controlled trial. Lancet Oncology. 2011, 12(11), 997-1003.

[30] Stummer W., Pichlmeier U., Meinel T., Wiestler O.D., Zanella F., Reulen H.-J., for the ALA-Glioma Study Group. Fluorescence-guided surgery with 5-aminolevulinic acid for resection of malignant glioma: a randomized controlled multicenter phase III trial. Lancet Oncology. 2006, 7(5), 392-401.

[31] Ojemann G., Ojemann J., Lettich E., Berger M. Cortical language localization in left, dominant hemisphere: an electrical stimulation mapping investigation in 117 patients. Journal of Neurosurgery. 1989, 71(3), 316-326.

[32] Sanai N., Mirzadeh Z., Berger M.S. Functional outcome after language mapping for glioma resection. New England Journal of Medicine. 2008, 358(1), 18-27.

[33] Mikuni N., Miyamoto S. Surgical treatment of glioma: extent of resection applying functional neurosurgery. Neurologia Medico-Chirurgica. 2010, 50(9), 720-726.

[34] Senft C., Forster M.-T., Bink A., Mittelbronn M., Franz K., Seifert V., Szelenyi A. Optimizing the extent of resection in eloquently located gliomas by combining intraoperative MRI guidance with intraoperative neurophysiological monitoring. Journal of Neuro-Oncology. 2012, 109(1), 81-90.

[35] Bello L., Galucci M., Fava M., Carrabba G., Giussani C., Acerbi F., Baratta P., Songa V., Conte V., Branca V., Stocchetti N., Papagno C., Gaini S.M. Intraoperative subcortical language tract mapping guides surgical removal of gliomas involving speech areas. Neurosurgery. 2007, 60(1), 67-82.

[36] Kim S.S., McCutcheon I.E., Suki D., Weinberg J.S., Sawaya R., Lang F.F., Ferson D., Heimberger A.B., DeMonte F., Prabhu S.S. Awake craniotomy for brain tumors near eloquent cortex: correlation of intraoperative cortical mapping with neurological outcomes in 309 consecutive patients. Neurosurgery. 2009, 64(5), 836-846.

[37] Otani N., Bjeljac M., Muroi C., Weniger D., Khan N., Wieser H.G., Curcic M., Yonekawa Y. Awake surgery for glioma resection in eloquent areas: Zurich's experience and review. Neurologia MedicoChirurgica. 2005, 45(10), 501-511.

[38] Suzuki T., Sakurai Y., Yoshimitsu K., Nambu K., Muragaki Y., Iseki H. Intraoperative multichannel audio-visual information recording and automatic surgical phase and incident detection. Conf Proc IEEE Eng Med Biol Soc. 2010, 1190-1193.

[39] Zamorano L., Li Q., Jain S., Kaur G. Robotics in neurosurgery: state of the art and future technological challenges. International Journal of Medical Robotics and Computer Assisted Surgery. 2004, 1(1), 7-22.

[40] Hongo K., Kobayashi S., Kakizawa Y., Koyama J., Goto T., Okudera H., Kan K., Fujie M.G., Iseki H., Takakura K. NeuRobot: telecontrolled micromanipulator system for minimally invasive microneurosurgery-preliminary results. Neurosurgery. 2002, 51(4), 985-988. 



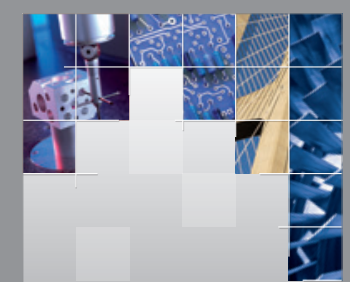

\section{Enfincering}
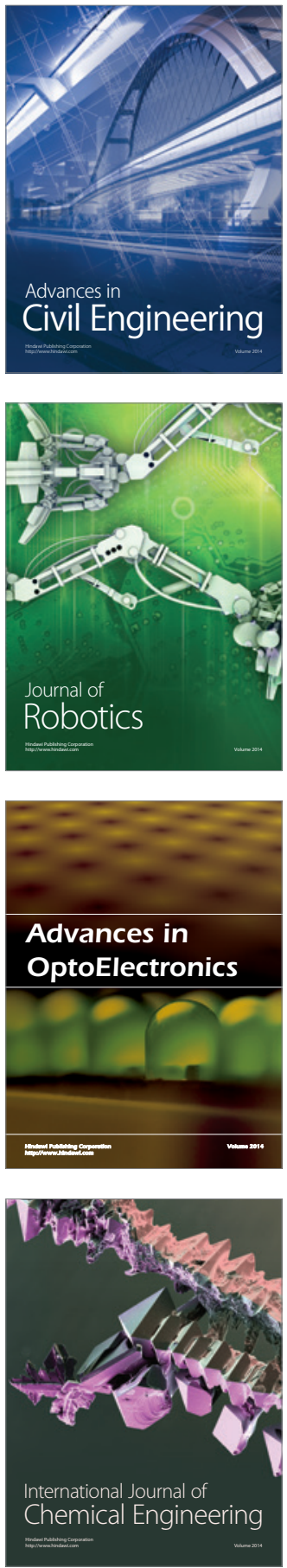

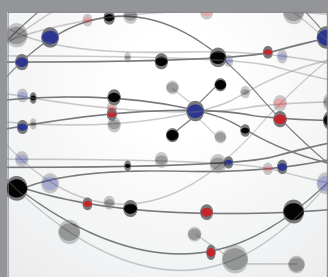

The Scientific World Journal

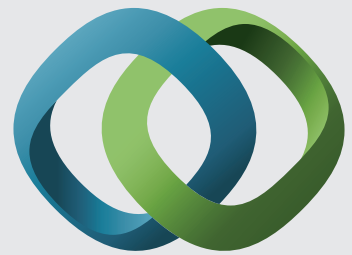

\section{Hindawi}

Submit your manuscripts at

http://www.hindawi.com
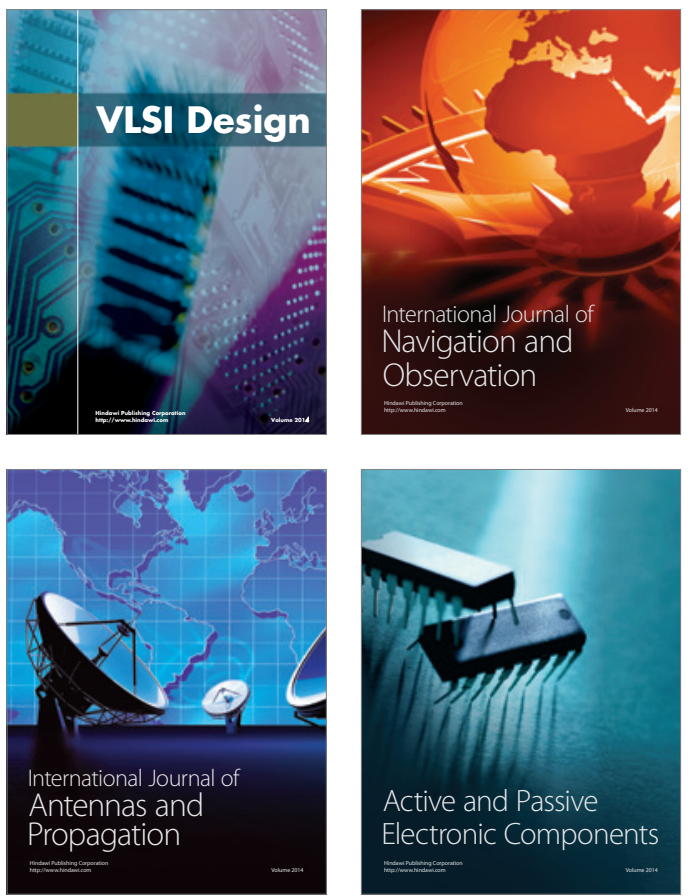
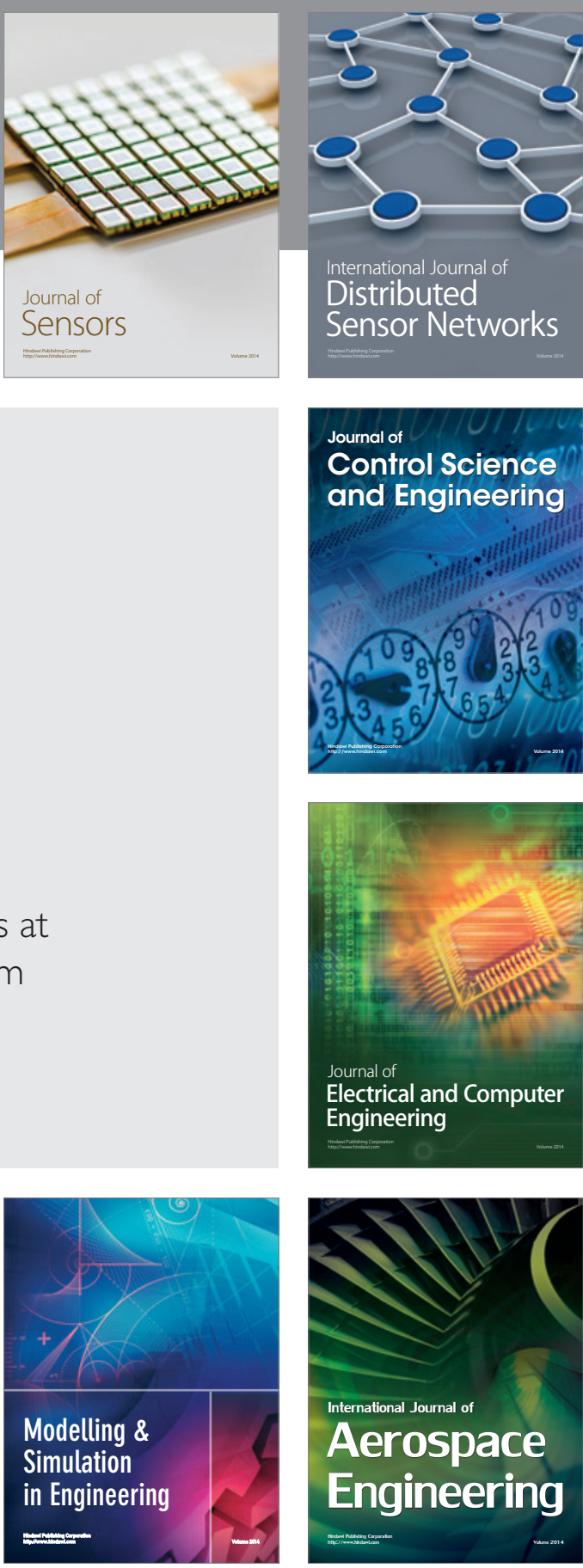

International Journal of

Distributed

Sensor Networks

Journal of

Control Science

and Engineering
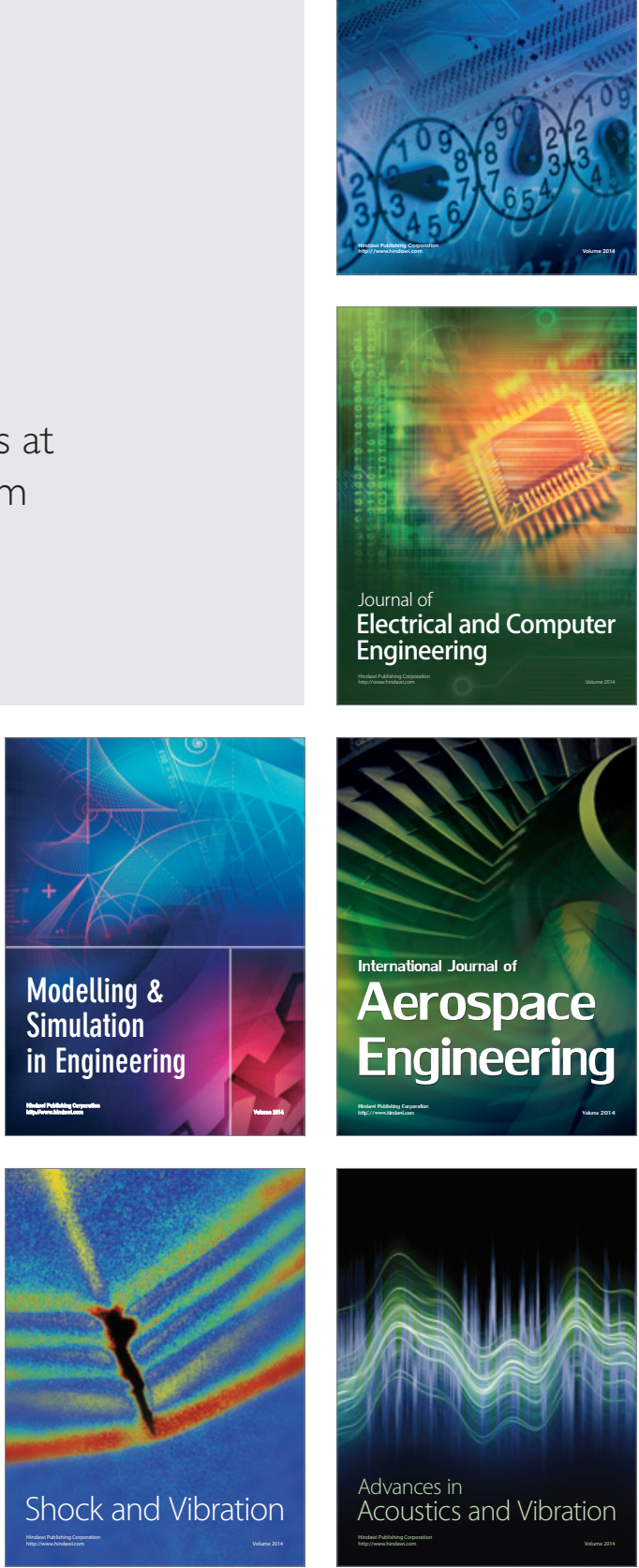